\title{
Some Algorithms for Prime Testing Using Generalized Lehmer Functions
}

\author{
By H. C. Williams and J. S. Judd
}

\begin{abstract}
Let $N$ be an odd integer thought to be prime. The properties of special functions which are generalizations of the functions of Lehmer (Ann. of Math., v. 31, 1930, pp. 419-448) are used to develop algorithms that produce information concerning the possible prime divisors of $N$. It is shown how the factors of $N \pm 1, N^{2}+1$, $N^{2} \pm N+1$, together with the factor bounds on these numbers, may all be used to calculate lower bounds for the possible prime divisors of $N$. Frequently, these bounds are large enough that $N$ may be shown to be prime.

These tests were implemented on an IBM/370-158 computer and run on the pseudoprime divisors of the first 385 Fibonacci and Lucas numbers.
\end{abstract}

1. Introduction. In Brillhart, Lehmer, and Selfridge [1], it was shown how an odd integer $N$, suspected to be prime, may be proved prime provided a sufficient number of factors of $N-1$ and/or $N+1$ have been determined. Later Williams and Judd [10] showed that if there were not enough factors of $N \pm 1$ known to prove the primality of $N$, the factors of $N^{2}+1$ could also be used. In an attempt, however, to demonstrate the primality of

$$
N=13484292549345009218015967701713491137426073107017330576389569
$$

the large (62 digits) pseudoprime factor of the Lucas* number $l_{368}$, we find

$$
\begin{aligned}
N-1 & =2^{6} \cdot 11 \cdot 17 \cdot 23 \cdot R_{1}, \\
N+1 & =2 \cdot 3 \cdot 5 \cdot 7^{2} \cdot 389 \cdot R_{2}, \\
N^{2}+1 & =2 \cdot 193 \cdot 37217 \cdot 1717117 \cdot R_{4},
\end{aligned}
$$

with each of $R_{1}, R_{2}, R_{4}$ being composite and having any prime divisor greater than $4 \times 10^{6}$. This is not a sufficient number of factors to prove $N$ a prime by using the tests of [1] and [10]; but, if we examine $N^{2}-N+1$, we find

$$
N^{2}-N+1=3 \cdot 109 \cdot 216757 \cdot 1339903 \cdot R_{6} .
$$

In this paper we will develop methods which allow the factors of $N \pm 1, N^{2}+1$, $N^{2} \pm N+1$ to be utilized in an attempt to show that $N$ is a prime. As was done in [10], we make use of the properties of the generalized Lehmer functions of Williams [9] in order to develop the theoretical background necessary for establishing these

Received October 7, 1975.

AMS (MOS) subject classifications (1970). Primary 10A25; Secondary 10A35.

Key words and phrases. Primality testing, generalized Lehmer functions, Fibonacci numbers, Lucas numbers.

*We use the usual notations $l_{n}$ and $f_{n}$ for the $n$th Lucas number and Fibonacci number, respectively. That is, $l_{0}=2, l_{1}=1, f_{0}=0, f_{1}=1, l_{n+1}=l_{n}+l_{n-1}, f_{n+1}=f_{n}+f_{n-1}$. 
algorithms. In the last two sections we discuss the results of a computer run on the numbers labelled pseudoprime in the table of factors of $l_{n}$ and $f_{n}$ in Jarden [2]. We also present several detailed examples.

It should be noted at this point that D. H. Lehmer [3] , [4] has previously considered the possibility of using factors of $N^{2}+N+1$ to demonstrate the primality of $N$. His technique, however, involves the use of Pierce's [6] functions; and it also requires that $N^{2}+N+1$ be completely factored.

2. The Function $C_{n}$. Let $f(x)$ be a polynomial

$$
x^{s}-P_{1} x^{s-1}+P_{2} x^{s-2}-\cdots+(-1)^{s} P_{s}
$$

with integer coefficients and $s$ distinct zeros $\rho_{1}, \rho_{2}, \ldots, \rho_{s}$.

Let $Q$ be an integer such that $\left(P_{1}, P_{2}, \ldots, P_{s}, Q\right)=1$; and let $\alpha_{i}, \beta_{i}(i=1$, $2, \ldots, s)$ be the zeros of $x^{2}-\rho_{i} x+Q(i=1,2, \ldots, s)$. Put

$$
\begin{gathered}
\delta=\left|\begin{array}{cccccc}
1 & \rho_{1} & \rho_{1}^{2} & \cdots & \cdot & \rho_{1}^{s-1} \\
1 & \rho_{2} & \rho_{2}^{2} & \cdot & \cdot & \rho_{2}^{s-1} \\
\cdot & \cdot & \cdot & \cdots & \cdot & \cdot \\
1 & \rho_{s} & \rho_{s}^{2} & \cdots & \cdot & \rho_{s}^{s-1}
\end{array}\right|, \\
\Delta=\delta^{2}, \quad E=f(2 \sqrt{Q}) f(-2 \sqrt{Q}), \\
v_{n}\left(\rho_{i}\right)=\alpha_{i}^{n}+\beta_{i}^{n} \quad(i=1,2, \ldots, s)
\end{gathered}
$$

and define $V_{j, n}(j=0,1,2, \ldots, s-1)$ as

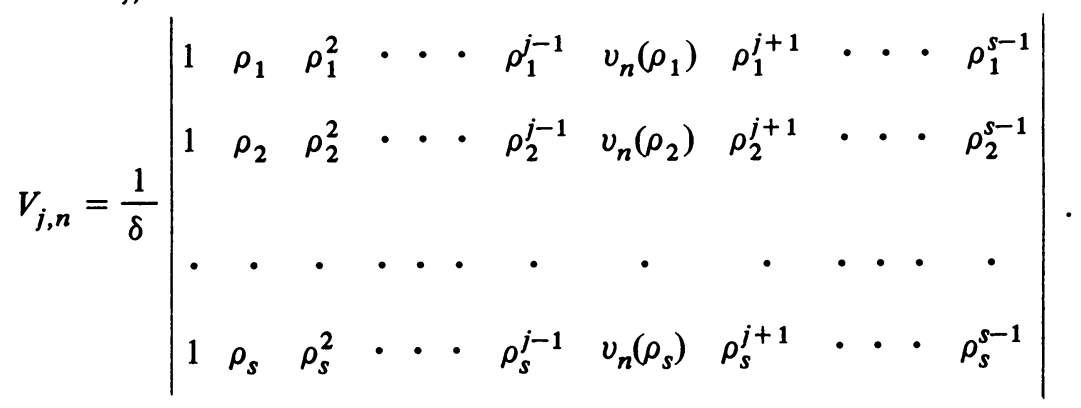

The function $C_{n}$ is then defined to be the greatest common divisor $\left(V_{1, n}, V_{2, n}\right.$, $\left.V_{3, n}, \ldots, V_{s-1, n}\right)$ of $V_{1, n}, V_{2, n}, V_{3, n}, \ldots, V_{s-1, n}$.

As we shall be most concerned in this paper with the case $s=3$, we conclude this section with some special properties of $V_{0, n}, V_{1, n}, V_{2, n}, C_{n}$ for $s=3$. We first note that

$$
\begin{aligned}
& \Delta=P_{1}^{2} P_{2}^{2}+18 P_{1} P_{2} P_{3}-4 P_{2}^{3}-4 P_{1}^{3} P_{3}-27 P_{3}^{2}, \\
& E=\left(P_{3}+4 Q P_{1}\right)^{2}-Q\left(2 P_{2}+8 Q\right)^{2} .
\end{aligned}
$$

The first few values for the functions $V_{0, n}, V_{1, n}$ and $V_{2, n}$ are given in the following table. 


\begin{tabular}{c|c|c|c|}
\hline$n$ & $V_{0, n}$ & $V_{1, n}$ & $V_{2, n}$ \\
\hline 0 & 2 & 0 & 0 \\
1 & 0 & 1 & 0 \\
2 & $-2 Q$ & 0 & 1 \\
3 & $P_{3}$ & $-P_{2}-3 Q$ & $P_{1}$ \\
4 & $P_{3} P_{1}+2 Q^{2}$ & $P_{3}-P_{1} P_{2}$ & $P_{1}^{2}-P_{2}-4 Q$ \\
5 & $P_{3} P_{1}^{2}-P_{2} P_{3}-5 Q P_{3}$ & $P_{2}^{2}-P_{2} P_{1}^{2}+P_{1} P_{3}+5 Q P_{2}+5 Q^{2}$ & $P_{1}^{3}-2 P_{1} P_{2}-5 Q P_{1}+P_{3}$ \\
\hline
\end{tabular}

Also, each of the functions $V_{0, n}, V_{1, n}$ and $V_{2, n}$ satisfies the recurrence

$$
\begin{aligned}
X_{n+6}= & P_{1} X_{n+5}-\left(P_{2}+3 Q\right) X_{n+4}+\left(2 P_{1} Q+P_{3}\right) X_{n+3} \\
& -\left(3 Q^{2}+Q P_{2}\right) X_{n+2}+P_{1} Q^{2} X_{n+1}-Q^{3} X_{n}
\end{aligned}
$$

and

$$
\begin{aligned}
& V_{0, n+1}=P_{3} V_{2, n}-Q V_{0, n-1}, \\
& V_{1, n+1}=V_{0, n}-P_{2} V_{2, n}-Q V_{1, n-1}, \\
& V_{2, n+1}=V_{1, n}+P_{1} V_{2, n}-Q V_{2, n-1} .
\end{aligned}
$$

If $N$ is any integer and $\left(N, Q P_{3}\right)=1$, find $M, S$ such that

$$
Q M \equiv P_{3} S \equiv 1 \quad(\bmod N)
$$

and put

$$
\begin{aligned}
& X_{k}= \begin{cases}S^{2} M^{k / 2} V_{0, k}, & k \text { even, } \\
S M^{(k+1) / 2} V_{0, k}, & k \text { odd }\end{cases} \\
& Y_{k}= \begin{cases}S^{2} M^{k / 2} V_{1, k}, & k \text { even, } \\
S M^{(k+1) / 2} V_{1, k}, & k \text { odd }\end{cases} \\
& Z_{k}= \begin{cases}S^{2} M^{k / 2} V_{2, k}, & k \text { even, } \\
S M^{(k+1) / 2} V_{2, k}, & k \text { odd. }\end{cases}
\end{aligned}
$$

Then,

$$
X_{2 m+1}=P_{3}\left(Y_{2 m+2}+Y_{2 m}\right)+P_{2} Z_{2 m+1},
$$


Also,

$$
\begin{aligned}
& Y_{2 m+1}=P_{3}\left(Z_{2 m+2}+Z_{2 m}\right)-P_{1} Z_{2 m+1} \\
& Z_{2 m+1}=X_{2 m+2}+X_{2 m} .
\end{aligned}
$$

$$
\begin{aligned}
& X_{2 m}=Q\left(X_{m}^{2}+2 Y_{m} Z_{m} P_{3}+P_{1} P_{3} Z_{m}^{2}\right)-2 S^{2}, \\
& Y_{2 m}=Q\left(2 X_{m} Y_{m}-2 P_{2} Y_{m} Z_{m}+\left(P_{3}-P_{1} P_{2}\right) Z_{m}^{2}\right), \\
& Z_{2 m}=Q\left(Y_{m}^{2}+2 Z_{m} X_{m}+2 P_{1} Y_{m} Z_{m}+\left(P_{1}^{2}-P_{2}\right) Z_{m}^{2}\right),
\end{aligned}
$$

when $m$ is odd. If $m$ is even, replace the $Q$ in these formulas by $P_{3}^{2}$. Using these formulas, we can evaluate $Y_{k}$, and $Z_{k}$ in $O(\log k)$ operations. Since $\left(Y_{k}, Z_{k}, N\right)=$ $\left(V_{1, k}, V_{2, k}, N\right)$, we see that this technique can be used for evaluating $\left(C_{k}, N\right)$.

3. Properties of $C_{n}$. In [9] several divisibility properties of $C_{n}$ are presented; for example, $C_{n} \mid C_{m n}$ if $n \mid m$. The following definition is also given.

Let $m$ be any integer such that $(m, Q)=1$ and let $C_{\tau_{0}}$ be the first term of the sequence

$$
C_{1}, C_{2}, C_{3}, \ldots, C_{n}, \ldots
$$

in which $m$ occurs as a factor. We define the increasing sequence of integers

$$
\tau_{0}, \tau_{1}, \tau_{2}, \ldots, \tau_{j}, \ldots
$$

by saying that $C_{\tau_{j}}$ is the first term of the sequence (*) such that $m \mid C_{\tau_{j}}$ and $\tau_{i} \nmid \tau_{j}$ $(i=0,1,2, \ldots, j-1)$. We call these $\tau$ 's the orders of apparition of $m$ and denote them by $\tau_{j}(m)$.

It is then demonstrated that if $(m, Q)=1$, then any order of apparition $\tau(m)$ must be a divisor of $2 \Phi(m)$, where $\Phi(m)$ is a rather complicated function which depends on $m, Q$, and the polynomial $f(x)$. When $f(x)$ is irreducible modulo a prime $p$ and $s$ is odd, we can obtain some special results about the orders of apparition of $p$. We first give some simple lemmas.

LEMMA 1. If $f(x)$ is irreducible modulo $p$, then $p \mid C_{n}$ if and only if $v_{n}\left(\rho^{*}\right) \in$ $\mathrm{GF}[p]$, where $\rho^{*}$ is a root of $f(x)=0$ in $\mathrm{GF}\left[\rho^{s}\right]$.

Proof. Let

$$
v_{n}\left(\rho^{*}\right)=\sum_{j=0}^{s-1} V_{j, n}^{*} \rho^{* j},
$$

where $V_{j, n}^{*} \in \operatorname{GF}[p](j=0,1,2, \ldots, s-1)$.

Now $p \mid C_{n}$ if and only if $V_{1, n}^{*}=V_{2, n}^{*}=V_{3, n}^{*}=\cdots=V_{s-1, n}^{*}=0$; thus, if $p \mid C_{n}, v_{n}\left(\rho^{*}\right) \in \mathrm{GF}[p]$. If $v_{n}\left(\rho^{*}\right) \in \mathrm{GF}[p]$, then

$$
A=\sum_{j=0}^{s-1} V_{j, n}^{*} \rho^{* j} \in \mathrm{GF}[p] .
$$

But, since $f(x)$ is irreducible modulo $p$, we must have

$$
V_{0, n}^{*}=A, \quad V_{1, n}^{*}=V_{2, n}^{*}=\cdots=V_{s-1, n}^{*}=0 ;
$$

hence, $P \mid C_{n}$. 
Lemma 2. Let $A, B \in K=\mathrm{GF}\left[p^{2 s}\right]$, where $A B \neq 0$ and $s$ is odd. If $A+B \in$ $\mathrm{GF}\left[p^{s}\right], A B \in \mathrm{GF}[p], A^{k}+B^{k} \in \mathrm{GF}[p]$ and $A^{m}+B^{m} \in \mathrm{GF}[p]$, then $A^{r}+B^{r} \in$ $\mathrm{GF}[p]$, where $m=q k+r$.

Proof. In $K$ we have

$$
A^{k p^{2}}=A^{k}, \quad B^{k p^{2}}=B^{k}, \quad A^{m p^{2}}=A^{m}, \quad B^{m p^{2}}=B^{m}
$$

hence,

$$
A^{q k+r}=A^{m p^{2}}=A^{q k p^{2}+r p^{2}}=A^{q k+r p^{2}}
$$

and $A^{r}=A^{r p^{2}}$, also $B^{r}=B^{r p^{2}}$. Since $A^{r}+B^{r} \in \mathrm{GF}\left[p^{s}\right]$, we have

$$
\left(A^{r}+B^{r}\right)^{p^{s}}=A^{r}+B^{r}, \quad\left(A^{r}+B^{r}\right)^{p^{2}}=A^{r}+B^{r} .
$$

Since $s$ is odd, it follows that $\left(A^{r}+B^{r}\right)^{p}=A^{r}+B^{r}$; and consequently, $A^{r}+B^{r} \in$ $\mathrm{GF}[p]$.

COROLlaRY. If the conditions of the lemma are true, then $A^{d}+B^{d} \in \mathrm{GF}[p]$, where $d=(k, m)$.

We are now able to prove the following

THEOREM. If $p \nmid 2 \Delta E Q$ and $f(x)$ is of odd degree $s$ and irreducible modulo $p$, then there is only one order of apparition $\tau$ of $p$ and $\tau \mid\left(p^{s}-\epsilon\right) /(p-\epsilon)$, where $\epsilon=$ $(E \mid p)$ (Legendre Symbol).

Proof. Let $\rho^{*}$ be a zero of $f(x)$ in GF $\left[p^{s}\right]$; then the other zeros are given by $\rho^{* p}, \rho^{* p^{2}}, \ldots, \rho^{* p^{s-1}}$ also $\rho^{* p^{s}}=\rho^{*}$. Let $\alpha^{*}, \beta^{*}$ be the two zeros of $x^{2}-\rho^{*} x+$ $Q$ in $\mathrm{GF}\left[p^{2 s}\right]$. We have

$$
v_{n}\left(\rho^{*}\right)=\alpha^{* n}+\beta^{* n}
$$

Now $\left(2 \alpha^{*}-\rho^{*}\right)^{2}=\rho^{* 2}-4 Q$; hence,

$$
\begin{aligned}
\left(2 \alpha^{*}-\rho^{*}\right)^{p^{s}-1} & =\left(\rho^{*^{2}}-4 Q\right)^{\left(p^{s}-1\right) / 2} \\
& =\left[\left(\rho^{*^{2}}-4 Q\right)^{p^{s-1}}\left(\rho^{*^{2}}-4 Q\right)^{p^{s-2}} \cdots\left(\rho^{*^{2}}-4 Q\right)\right]^{(p-1) / 2} \\
& =\left[\left(\left(\rho^{* p^{s-1}}\right)^{2}-4 Q\right)\left(\left(\rho^{* p^{s-2}}\right)^{2}-4 Q\right) \cdots\left(\rho^{*^{2}}-4 Q\right)\right]^{(p-1) / 2} \\
& =E^{(p-1) / 2}=\epsilon .
\end{aligned}
$$

We see that $\left(2 \alpha^{*}-\rho^{*}\right)^{s}=\epsilon\left(2 \alpha^{*}-\rho^{*}\right)$ and

$$
\alpha^{* p^{s}}= \begin{cases}\alpha^{*} & \text { if } \epsilon=1, \\ \beta^{*} & \text { if } \epsilon=-1\end{cases}
$$

Putting $k=\left(p^{s}-\epsilon\right) /(p-\epsilon)$, we get

$$
\begin{aligned}
v_{k}\left(\rho^{*}\right)^{p} & =\alpha^{* k p}+\beta^{* k p}=\alpha^{*(p-\epsilon) k+\epsilon k}+\beta^{*(p-\epsilon) k+\epsilon k} \\
& =\alpha^{*\left(p^{s}-\epsilon\right)} \alpha^{* \epsilon k}+\beta^{*\left(p^{s}-\epsilon\right)} \beta^{* \epsilon k}=v_{k}\left(\rho^{*}\right)
\end{aligned}
$$

It follows that $p \mid C_{k}$. 
We have shown that there exists one order of apparition $\tau$ of $p$ and that $\tau \mid k$. Suppose there exists a second order of apparition $\tau_{1}$ of $p$. We have

$$
\alpha^{* \tau}+\beta^{* \tau} \in \mathrm{GF}[p], \quad \alpha^{* \tau} 1+\beta^{* \tau} 1 \in \mathrm{GF}[p]
$$

hence,

$$
\alpha^{* d}+\beta^{* d} \in \mathrm{GF}[p],
$$

where $d=\left(\tau, \tau_{1}\right)$. Now $d<\tau, d \mid \tau$ and $p \mid C_{d}$. By definition of $\tau$ this is impossible and the theorem is proved.

Let $f(x)$ be a polynomial of odd degree $s$ such that, for any prime $p \nmid \Delta, f(x)$ is either irreducible modulo $p$ or completely reducible. For example, the cyclotomic period equation [5] is such a polynomial. (For $s=3$ the necessary and sufficient condition for $f(x)$ to be this type of polynomial is that $\Delta$ be a perfect square.) For $C_{n}$ defined for such an $f$, we define

$$
\psi(p)= \begin{cases}\left(p^{s}-\epsilon\right) /(p-\epsilon) & \text { if } f(x) \text { is irreducible }(\bmod p), \\ 2\left[p-\eta_{1}, p-\eta_{2}, p-\eta_{3}, \ldots, p-\eta_{s}\right] & \text { if } f(x) \text { is reducible }(\bmod p),\end{cases}
$$

where $\eta_{i}=\left(r_{i}^{2}-4 Q \mid p\right)$ and $r_{1}, r_{2}, r_{3}, \ldots, r_{s}$ are the $s$ roots of $f(x) \equiv 0(\bmod p)$.

With this definition of $f$ and $C_{n}$ we have the following two theorems.

THEOREM. If $p$ is a prime and $(p, 2 \Delta E Q)=1$, then there exists at least one order of apparition of $p$. Further, if $\tau_{j}(p)$ is any order of apparition of $p$, then $\tau_{j}(p) \mid \psi(p)$

Proof. This follows easily from the Law of Apparition of [9] and the previous theorem.

THEOREM. Let $(N, 2 \Delta Q E)=1$ and $N \mid C_{m}$. If $q$ is any prime divisor of $m$ and $N \nmid C_{m / q}$, then any prime divisor $p$ of $N$ which does not divide $C_{m / q}$ must satisfy the congruence

$$
\psi(p) \equiv 0 \quad\left(\bmod q^{\alpha}\right)
$$

where $q^{\alpha} \| m$.

Proof. Let $\tau$ be an order of apparition of $p$ such that $\tau \mid m$. Clearly, since $p \mid C_{m}$, such a $\tau$ must exist. Now $p \nmid C_{m / q}$; hence, $\tau \nmid m / q$; and consequently, $q^{\alpha} \mid \tau$. Since $\tau(p) \mid \psi(p)$, we have

$$
\psi(p) \equiv 0\left(\bmod q^{\alpha}\right)
$$

4. The Sequences $\left\{C_{n}^{(i)}\right\}$. In the remainder of this paper we will consider $s$ to have the value 3 .

Let $N$ be an integer which we wish to test for primality. Select a prime $P$ such that $P \equiv 1(\bmod 3)$ and $(N \mid P)_{3} \neq 1$, and let $4 P=S^{2}+27 T^{2}$, where $S \equiv 1(\bmod 3)$. Then, if $N$ is a prime and $(N, P T)=1, x^{3}-a x-b$, where $a=3 P, b=P S$, is irreducible modulo $N$. Let $G$ be a fixed integer and put $\theta=(G \mid N)$, where $|\theta|=1$.

For any three integers $h_{i}, k_{i}, l_{i}$, put 


$$
\begin{gathered}
m_{1}=h_{i}^{2}+2 b l_{i} k_{i}-G, \quad m_{2}=b l_{i}^{2}+2 h_{i} k_{i}+2 a k_{i} l_{i}, \\
m_{3}=k_{i}^{2}+a l_{i}^{2}+2 h_{i} l_{i}, \\
d_{1}=m_{1}^{2}+2 a m_{3} m_{1}+a^{2} m_{3}^{2}-a m_{2}^{2}-b m_{2} m_{3}, \quad d_{2}=b m_{3}^{2}-m_{1} m_{2}, \\
d_{3}=m_{2}^{2}-m_{1} m_{3}-a m_{3}^{2} .
\end{gathered}
$$

Let

$$
\begin{gathered}
R=d_{1} m_{1}+b d_{2} m_{3}+b d_{3} m_{2}, \\
A=4 G d_{1}+2 R, \quad B=4 G d_{2}, \quad C=4 G d_{3} .
\end{gathered}
$$

We define the sequence $C_{n}^{(i)}$ by using the parameters below:

$$
\begin{gathered}
P_{1}^{(i)}=3 A+2 a C, \quad P_{2}^{(i)}=3 A^{2}+4 a A C-a B^{2}-3 b B C+a^{2} C^{2}, \\
P_{3}^{(i)}=A^{3}+b B^{3}+b^{2} C^{3}-3 b A B C-a A B^{2}-a b B C^{2}+a^{2} A C^{2}+2 a A^{2} C, \\
Q^{(i)}=R^{2} .
\end{gathered}
$$

If $\sigma_{1}, \sigma_{2}, \sigma_{3}$ are the zeros of $x^{3}-a x-b$, we see that $\rho_{j}=A+B \sigma_{j}+C \sigma_{j}^{2}$ $(j=1,2,3)$ are the three zeros of

$$
f(x)=x^{3}-P_{1}^{(i)} x^{2}+P_{2}^{(i)} x-P_{3}^{(i)} .
$$

Now let $\sigma$ be any one of the three zeros $\sigma_{1}, \sigma_{2}, \sigma_{3}$ and put

$$
\begin{gathered}
\rho=A+B \sigma+C \sigma^{2}, \quad X(\sigma)=h_{i}+k_{i} \sigma+l_{i} \sigma^{2}, \\
Y(\sigma)=d_{1}+d_{2} \sigma+d_{3} \sigma^{2} .
\end{gathered}
$$

Then

$$
\begin{gathered}
m_{1}+m_{2} \sigma+m_{3} \sigma^{2}=(X(\sigma))^{2}-G \\
R=Y(\sigma)\left(m_{1}+m_{2} \sigma+m_{3} \sigma^{2}\right)=Y(\sigma)\left((X(\sigma))^{2}-G\right) \\
\rho=4 Y(\sigma) G+2 R .
\end{gathered}
$$

It follows that

$$
\rho^{2}-4 Q=[4 Y(\sigma) X(\sigma)]^{2} G .
$$

Since $E^{(i)}=\left(\rho_{1}^{2}-4 Q\right)\left(\rho_{2}^{2}-4 Q\right)\left(\rho_{3}^{2}-4 Q\right)$, we have $E^{(i)}=V^{2} G$, where $V=$ $4^{3} G Y\left(\sigma_{1}\right) Y\left(\sigma_{2}\right) Y\left(\sigma_{3}\right) X\left(\sigma_{1}\right) X\left(\sigma_{2}\right) X\left(\sigma_{3}\right)$.

Also,

$$
\begin{aligned}
\Delta^{(i)} & =\left(B^{3}-a B C^{2}-b C^{3}\right)^{2}\left|\begin{array}{lll}
1 & \sigma_{1} & \sigma_{1}^{2} \\
1 & \sigma_{2} & \sigma_{2}^{2} \\
1 & \sigma_{3} & \sigma_{3}^{2}
\end{array}\right|^{2} \\
& =3^{6}\left(B^{3}-a B C^{2}-b C^{3}\right)^{2}(P T)^{2} .
\end{aligned}
$$


Thus, if $p$ is any prime such that $\left(p, 2 \Delta^{(i)} E^{(i)} Q^{(i)}\right)=1$, then $\left(\Delta^{(i)} \mid p\right)=1,\left(E^{(i)} \mid p\right)=$ $(G \mid p)=\theta(p)$ and if $f(x)$ is reducible, $\eta_{i}=\theta(p)(i=1,2,3)$. We see that these are independent of the values of $h_{i}, k_{i}, l_{i}$. Also, if $x^{3}-a x-b$ is irreducible $(\bmod p)$, then so is $f(x)$; thus, $\psi(p)$ is always the same for any sequence $\left\{C_{n}^{(i)}\right\}$. Also $\psi(p)=$ $2(p-\theta(p))$ when $f(x)$ is reducible modulo $p$.

5. Some Criteria for Primality. Denote by $F_{3}$ the completely factored part of $N^{2}+N+1$ and by $F_{6}$ the completely factored part of $N^{2}-N+1$. Then $N^{2}+$ $N+1=F_{3} R_{3}, N^{2}-N+1=F_{6} R_{6}$, where $\left(R_{3}, F_{3}\right)=\left(F_{6}, R_{6}\right)=1$. For $\theta=1$, put

(1) For each prime $q \mid F_{3}$, there exists some $h_{i}, k_{i}, l_{i}$ such that $\left(N, \Delta^{(i)} E^{(i)} Q^{(i)}\right)$ $=1$ for the sequence $\left\{C_{n}^{(i)}\right\}$,

$$
N \mid C_{N^{2}+N+1}^{(i)} \text { and } \quad\left(C_{\left(N^{2}+N+1\right) / q}^{(i)}, N\right)=1 .
$$

(2) For some $h_{i}, k_{i}, l_{i}$ such that $\left(N, \Delta^{(i)} E^{(i)} Q^{(i)}\right)=1$ for the sequence $\left\{C_{n}^{(i)}\right\}$, we have

$$
\left.\underset{N^{2}+N+1}{N \mid C^{(i)}} \text { and } \underset{\left(N^{2}+N+1\right) / R_{3}}{\left(C^{(i)}\right.}, N\right)=1 .
$$

For $\theta=-1$, put

(3) For each prime $q \mid F_{6}$, there exists some $h_{i}, k_{i}$, $l_{i}$ such that $\left(N, E^{(i)} \Delta^{(i)} Q^{(i)}\right)$ $=1$ for the sequence $\left\{C_{n}^{(i)}\right\}$,

$$
N \mid C_{N^{2}-N+1}^{(i)} \text { and } \quad\left(C_{\left(N^{2}-N+1\right) / q}^{(i)}, N\right)=1 .
$$

(4) For some $h_{i}, k_{i}, l_{i}$ such that $\left(N, \Delta^{(i)} E^{(i)} Q^{(i)}\right)=1$ for the sequence $\left\{C_{n}^{(i)}\right\}$, we have

$$
\left.\underset{N^{2}-N+1}{N \mid C^{(i)}} \text { and } \quad \underset{\left(N^{2}-N+1\right) / R_{6}}{(i)}, N\right)=1 .
$$

It should be noted that if $N \nmid C_{N^{2}}^{(i)}+\theta N+1$, then $N$ is composite.

We are now able to prove some theorems which give some information about possible prime factors of $N$ should any of (1), (2), (3), or (4) be true.

THEOREM. If $\theta=1,(1)$ is true, and $p$ is any prime divisor of $N$, then

$$
\psi(p) \equiv 0 \quad\left(\bmod F_{3}\right) .
$$

Proof. Since the value of $\psi(p)$ is the same for any of the sequences $\left\{C_{n}^{(i)}\right\}$ $(i=1,2, \ldots)$, it follows that if $q$ is any prime divisor of $F_{3}$ and (1) is true, then $q^{\nu} \mid \psi(p)$, where $q^{\nu} \| F_{3}$; hence $F_{3} \mid \psi(p)$.

THEOREM. If $\theta=1,(2)$ is true, and $p$ is any prime divisor of $N$, then

$$
\psi(p) \equiv 0 \quad(\bmod q)
$$

where $q$ is some prime divisor of $R_{3}$ depending on $p$.

Proof. Let $\tau=\tau(p)$ be an order of apparition of $p$ such that $\tau \mid N^{2}+N+1$; then $\tau \nmid F_{3}$; and consequently, $\left(R_{3}, \tau\right)>1$. Thus there must exist a prime $q$ such that $q \mid R_{3}$ and $q \mid \tau$. Since $\tau \mid \psi(p)$, the theorem follows. 
THEOREM. If $\theta=-1$ and (3) is true, and $p$ is any prime divisor of $N$, then

$$
\psi(p) \equiv 0 \quad\left(\bmod F_{6}\right)
$$

THEOREM. If $\theta=-1,(4)$ is true and $p$ is any prime divisor of $N$, then

$$
\psi(p) \equiv 0 \quad(\bmod q)
$$

where $q$ is some prime divisor of $R_{6}$ depending on $p$.

The theorems requiring the truth of either (2) or (4) are unfortunately not as useful here as their analogues in [1] or even [10]; however, we will show in a later section how these theorems can occasionally be useful.

We conclude this section with two results which allow us to demonstrate the primality of $N$ when either $N^{2}+N+1$ or $N^{2}-N+1$ is sufficiently factored.

THEOREM. If $\theta=1,(1)$ is true, $N$ is not a perfect square and $F_{3}>N^{2 / 3}>36$, then $N$ is a prime.

Proof. Suppose $N=p_{1} p_{2} p_{3} a$ and $a$ is any positive integer. Since

$$
\psi\left(p_{i}\right) \equiv 0 \quad\left(\bmod F_{3}\right)
$$

and $\psi\left(p_{i}\right)=p_{i}^{2} \pm p_{i}+1$ or $p_{i} \pm 1$, we have $p_{i}>\sqrt{F_{3}}-1$. If $F_{3} \mid p_{i} \pm 1$, then $p_{i}>$ $F_{3}-1>\sqrt{3} \sqrt{F_{3}}-1$. Since $N$ is not a perfect cube there must be at least two distinct prime divisors $p_{1}$ and $p_{2}$. If $F_{3} \mid p_{1}^{2} \pm p_{1}+1$ and $F_{3} \mid p_{2}^{2} \pm p_{2}+1$, then for one of these $p$ 's, say $p_{1}$, it must be true that

$$
p_{1}^{2} \pm p_{1}+1 \geqslant 3 F_{3}
$$

Thus, we have

$$
N=p_{1} p_{2} p_{3} a>\left(\sqrt{F}_{3}-1\right)^{2}\left(\sqrt{3} \sqrt{F_{3}}-1\right)>F_{3}^{3 / 2},
$$

which is impossible.

Hence, if $N$ is not a prime, it must be the product of two primes $p_{1}, p_{2}$.

Now $\left(p_{1} p_{2} \mid P\right)_{3} \neq 1$ and $\left(G \mid p_{1} p_{2}\right)=1$; hence $\theta\left(p_{1}\right)=\theta\left(p_{2}\right)=\epsilon$, say. If $\left(p_{1} \mid P\right)_{3}=1$, then $\left(p_{2} \mid P\right)_{3} \neq 1$ and

$$
p_{1} \equiv \epsilon \quad\left(\bmod F_{3}\right) \quad \text { and } \quad p_{2}^{2}+\epsilon p_{2}+1 \equiv 0 \quad\left(\bmod F_{3}\right)
$$

We have

$$
p_{1}>2 F_{3}-1 \text { and } p_{2}>\sqrt{F_{3}}-1
$$

consequently,

$$
N=p_{1} p_{2}>\left(F_{3}\right)^{3 / 2}
$$

If $\left(p_{1} \mid P\right)_{3} \neq 1,\left(p_{2} \mid P\right)_{3} \neq 1$,

$$
p_{1}^{2}+\epsilon p_{1}+1 \equiv p_{2}^{2}+\epsilon p_{2}+1=p_{1}^{2} p_{2}^{2}+p_{1} p_{2}+1 \equiv 0 \quad\left(\bmod F_{3}\right)
$$

It follows that

$$
\left(p_{1}-p_{2}\right)\left(p_{1}+p_{2}+\epsilon\right) \equiv 0 \quad\left(\bmod F_{3}\right)
$$


If $q \mid F_{3}$ and $q \nmid p_{1}-p_{2}$, then $q \mid p_{1}+p_{2}+\epsilon$ and

$$
p_{1}^{2} \equiv \epsilon p_{2}, \quad p_{2}^{2} \equiv \epsilon p_{1} \quad(\bmod q)
$$

hence,

$$
q \mid 2 p_{1} p_{2}+1 \text { and } q \mid 3
$$

Thus,

$$
p_{1} \equiv p_{2} \quad\left(\bmod \bar{F}_{3}\right)
$$

where**

$$
\bar{F}_{3}= \begin{cases}F_{3} / 3 & \text { if } 3 \mid F_{3}, \\ F_{3} & \text { otherwise. }\end{cases}
$$

If $3 \mid F_{3}$, then $p_{1}^{2}+\epsilon p_{1}+1 \equiv 0(\bmod 3)$ and $p_{1} \equiv \epsilon(\bmod 3)$, also $p_{2} \equiv \epsilon(\bmod 3)$.

Since $\left(3, \bar{F}_{3}\right)=1$, we have

$$
p_{1} \equiv p_{2} \quad\left(\bmod F_{3}\right)
$$

Since $p_{1} \neq p_{2}$, we have $p_{1}>p_{2}$ and

$$
p_{1}=p_{2}+2 k F_{3} \text { and } p_{1}>2 F_{3}+1 \text {. }
$$

Hence

$$
N=p_{1} p_{2}>\left(2 F_{3}+1\right)\left(\sqrt{F}_{3}-1\right)>F_{3}^{3 / 2}
$$

Thus $N$ is a prime.

THEOREM. If $\theta=-1,(3)$ is true and $F_{6}>N^{2 / 3}>36$, then $N$ is a prime.

6. Prime Testing. Let $F_{1}$ be the completely factored part of $N-1, F_{2}$ be the completely factored part of $N+1$, and $F_{4}$ be the completely factored part of $N^{2}+1$. Put

$$
\begin{array}{rcc}
R_{1}=(N-1) / F_{1}, & R_{2}=(N+1) / F_{2}, & R_{4}=\left(N^{2}+1\right) / F_{4}, \\
\bar{F}_{1}=F_{1} / 2, & \bar{F}_{2}=F_{2} / 2, & \bar{F}_{4}=F_{4} / 2, \\
R_{2}=r+S \bar{F}_{1}, & 0 \leqslant r<\bar{F}_{1}, \\
2 R_{1} R_{2} \equiv s \quad\left(\bmod \bar{F}_{4}\right), & \\
S+s N \equiv t \quad\left(\bmod \bar{F}_{4}\right), & 0<s, t<\bar{F}_{4}, \\
\mu_{1}=-1+r F_{2}+t F_{1} \bar{F}_{2}, & \mu_{2}=1+s F_{1} \bar{F}_{2} .
\end{array}
$$

In [10] it was shown that if $C$ and $D$ are selected such that

**We also define

$$
\bar{F}_{6}= \begin{cases}F_{6} / 3 & \text { if } 3 \mid F_{6} \\ F_{6} & \text { otherwise }\end{cases}
$$




$$
(D \mid N)=\left(C^{2}-16 D \mid N\right)=-1,
$$

tests can be developed for demonstrating the primality of $N$. For very large $N$ we sometimes are unable to demonstrate the primality of $N$ but can use a result (Theorem 6) of [10] to show that $N$ is either a prime or $N=p_{1} p_{2}$, where $p_{1}$ and $p_{2}$ are primes,

$$
p_{1}=\mu_{1}+m_{1} F_{1} \bar{F}_{2} \bar{F}_{4}, \quad p_{2}=\mu_{2}+m_{2} F_{1} \bar{F}_{2} \bar{F}_{4},
$$

and $\left(D \mid p_{2}\right)=+1,\left(D \mid p_{1}\right)=-1,\left(C^{2}-16 D \mid p_{2}\right)=+1,\left(C^{2}-16 D \mid p_{1}\right)=-1$. (We call a prime $p$ such that $(D \mid p)=\left(C^{2}-16 D \mid p\right)=-1$ a prime of the first kind; otherwise, we say it is a prime of the second kind [10].) When this occurs, the tests (1) and (3) can be used to attempt to show the primality of $N$.

If we select $G$ such that $G \equiv u^{2}(\bmod N)$, then $(G \mid N)=+1$; and if $N$ is the product of the two primes $p_{1}, p_{2}$, then $\left(G \mid p_{1}\right)=\left(G \mid p_{2}\right)=1$. If (1) is true, we have three possible cases.

Case 1. $\left(p_{1} \mid P\right)_{3}=1,\left(p_{2} \mid P\right)_{3} \neq 1$. Here

$$
p_{1} \equiv 1\left(\bmod F_{3}\right), \quad p_{2} \equiv N\left(\bmod F_{3}\right) \text {. }
$$

Case 2. $\left(p_{1} \mid P\right)_{3} \neq 1,\left(p_{2} \mid P\right)_{3}=1$. Here

$$
p_{2} \equiv 1 \quad\left(\bmod F_{3}\right), \quad p_{1} \equiv N\left(\bmod F_{3}\right) .
$$

Case 3. $\left(p_{1} \mid P\right)_{3} \neq 1,\left(p_{2} \mid P\right)_{3} \neq 1$. Here

$$
\begin{gathered}
p_{1} \equiv p_{2} \quad\left(\bmod F_{3}\right) \\
p_{1}^{2} \equiv p_{2}^{2} \equiv N \quad\left(\bmod F_{3}\right) \quad \text { and } \quad p_{1}^{2}+p_{1}+1 \equiv p_{2}^{2}+p_{2}+1 \equiv 0 \quad\left(\bmod F_{3}\right)
\end{gathered}
$$

hence,

$$
p_{1} \equiv p_{2} \equiv-N-1 \quad\left(\bmod F_{3}\right) .
$$

If $H F_{1} \bar{F}_{2} \bar{F}_{4} \equiv 1\left(\bmod \bar{F}_{3}\right)$, we see that we must have

$$
m_{1} \equiv\left(1-\mu_{1}\right) H, \quad m_{2} \equiv\left(N-\mu_{2}\right) H \quad\left(\bmod \bar{F}_{3}\right)
$$

or

(a)

$$
m_{1} \equiv\left(N-\mu_{1}\right) H, \quad m_{2} \equiv\left(1-\mu_{2}\right) H \quad\left(\bmod \bar{F}_{3}\right),
$$

or

$$
m_{1} \equiv\left(-N-1-\mu_{1}\right) H, \quad m_{2} \equiv\left(-N-1-\mu_{2}\right) H \quad\left(\bmod \bar{F}_{3}\right) .
$$

If we select $G$ such that $G \equiv u^{2}\left(C^{2}-16 D\right)(\bmod N)$, then $(G \mid N)=-1$; and if $N$ is the product of the two primes $p_{1}, p_{2}$, then $\left(G \mid p_{2}\right)=+1,\left(G \mid p_{1}\right)=-1$; and if (3) is true, we again have three possible cases.

Case 1. $\left(p_{1} \mid P\right)_{3}=1,\left(p_{2} \mid P\right)_{3} \neq 1$. Here

$$
p_{1} \equiv-1 \quad\left(\bmod F_{6}\right), \quad p_{2} \equiv-N\left(\bmod F_{6}\right)
$$


Case 2. $\left(p_{1} \mid P\right)_{3} \neq 1,\left(p_{2} \mid P\right)_{3}=1$. Here

$$
p_{2} \equiv 1 \quad\left(\bmod F_{6}\right), \quad p_{1} \equiv N \quad\left(\bmod F_{6}\right)
$$

Case 3. $\left(p_{1} \mid P\right)_{3} \neq 1,\left(p_{2} \mid P\right)_{3} \neq 1$. In this case

$$
p_{1} \equiv-p_{2} \quad\left(\bmod F_{6}\right), \quad p_{1}^{2} \equiv p_{2}^{2} \equiv-N \quad\left(\bmod F_{6}\right)
$$

and

$$
p_{2}^{2}+p_{2}+1 \equiv p_{1}^{2}-p_{1}+1 \equiv 0 \quad\left(\bmod F_{6}\right)
$$

hence,

$$
p_{2} \equiv N-1 \quad\left(\bmod F_{6}\right) \text { and } p_{1} \equiv-N+1 \quad\left(\bmod F_{6}\right) \text {. }
$$

If $H^{\prime} F_{1} \bar{F}_{2} \bar{F}_{4} \equiv 1\left(\bmod \bar{F}_{6}\right)$, we see that we must have

$$
m_{1} \equiv\left(-1-\mu_{1}\right) H^{\prime}, \quad m_{2} \equiv\left(-N-\mu_{2}\right) H^{\prime} \quad\left(\bmod \bar{F}_{6}\right),
$$

or

(b)

$$
m_{1} \equiv\left(N-\mu_{1}\right) H^{\prime}, \quad \quad m_{2} \equiv\left(1-\mu_{2}\right) H^{\prime} \quad\left(\bmod \bar{F}_{6}\right),
$$

or

$$
m_{1} \equiv\left(-N+1-\mu_{1}\right) H^{\prime}, \quad m_{2} \equiv\left(N-1-\mu_{2}\right) H^{\prime} \quad\left(\bmod \bar{F}_{6}\right) .
$$

By using (a) or (b) or both, we can often increase the possible size of $m_{1}$ and $m_{2}$ to the point where we get $p_{1} p_{2}>N$; when this occurs we have proved $N$ a prime.

If by using the tests of [10] we are unable to show that $N$ is either prime or the product of two primes, we can use the tests (1) and (3) of this paper to increase $M_{3}$, the minimum size of a prime divisor of the first kind of $N$. This can be done by finding all the positive solutions $S_{1}, S_{2}, S_{3}, \ldots, S_{n}$ which are less than $K=$ $F_{1} \bar{F}_{2} \bar{F}_{3} \bar{F}_{4} \bar{F}_{6}$ of the system

$$
Z \equiv 1 \quad\left(\bmod F_{1} \bar{F}_{3}\right), \quad Z \equiv-1 \quad\left(\bmod F_{2} \bar{F}_{6}\right), \quad Z^{2} \equiv-1 \quad\left(\bmod F_{4}\right),
$$

and all the positive solutions $S_{1}^{\prime}, S_{2}^{\prime}, S_{3}^{\prime}, \ldots, S_{k}^{\prime}$, which are less than $K$ of the system

$$
\begin{gathered}
Z \equiv 1 \quad\left(\bmod F_{1}\right), \quad Z \equiv-1 \quad\left(\bmod F_{2}\right), \quad Z^{2} \equiv-1 \quad\left(\bmod F_{4}\right), \\
Z^{2}+Z+1 \equiv 0 \quad\left(\bmod F_{3}\right), \quad Z^{2}-Z+1 \equiv 0 \quad\left(\bmod F_{6}\right) .
\end{gathered}
$$

If $S=\min \left\{S_{1}, S_{2}, S_{3}, \ldots, S_{n}, S_{1}^{\prime}, S_{2}^{\prime}, S_{3}^{\prime}, \ldots, S_{k}^{\prime}\right\}$ and none of $S_{1}, S_{2}, S_{3}, \ldots$, $S_{n}, S_{1}^{\prime}, S_{2}^{\prime}, S_{3}^{\prime}, \ldots, S_{k}^{\prime}$ is a divisor of $N$, then, if $G$ is defined as above and (1) and (3) are both true, any prime of the first kind which divides $N$ must exceed $S+K$. Thus, $M_{3}$ must exceed $S+K$; and this is usually an increase in the previous size of $M_{3}$ as determined by the methods of [10].

Other methods which utilize the tests (1), (2), (3), or (4) can also be devised for proving primality. Some of these will be discussed with respect to certain examples in a later section. 
7. Some Computational Results. A computer program similar in design to that of Selfridge and Wunderlich [8] was written for an IBM/370-158 computer. This program incorporated the tests of [1], [10] and those of the present work (including the selection of $G$ as presented in Section 6) as a means of proving a given number $N$ prime. This program attempts to factor $N-1, N+1, N^{2}+1, N^{2}+N+1, N^{2}-$ $N+1$ by utilizing, as test divisors, all the primes less than a certain factor bound $B$. The method described by Wunderlich and Selfridge [11] was used in this particular program segment.

If, after the factoring, sufficient information is available to prove $N$ a prime, the required final tests are executed. If insufficient information is available, Pollard's [7] method is used to attempt to factor $R_{1}, R_{2}, R_{4}$. If this produces enough additional factors, the final tests are executed.

Should the computer still not have enough information to execute the final tests, the algorithm of [8] is used when either $R_{1}$ or $R_{2}$ is a pseudoprime. That is, the computer attempts to prove the pseudoprime a prime, or, failing that, attempts to increase the appropriate factor bound. (See [1, p. 627].)

This program was run on all the pseudoprimes listed in the factorization tables of $l_{n}$ and $f_{n}$ in [2]. Of the seventy-nine pseudoprimes, forty are easily found to be prime by using only the tests of [1] and $B=5 \times 10^{5}$. Two of the remaining numbers (the pseudoprime divisors of $f_{331}$ and $f_{353}$ ) have been discussed in [10]. The remaining thirty-seven are also all prime, and the techniques needed to demonstrate the primality of each one are described in Table 1.

In the first column of Table 1, we denote by $N_{n}$ the large pseudoprime factor of $l_{n}$ and by $\bar{N}_{n}$ the large pseudoprime factor of $f_{n}$. In the second column we give the number of digits of the pseudoprime in the first column; in the third column we give the value of $B$ the program used. When no entry appears in this column, $B=5$ $\times 10^{5}$. In the fourth column the final tests needed to prove $N_{n}$ or $\bar{N}_{n}$ a prime are given. These are presented as [1] to indicate that only the tests of [1] were needed; [1], [10] to indicate that the tests of both [1] and [10] were needed; and [1], [10], PW to indicate that the tests of [1], [10] and those of the present work were needed. Finally, in the fifth column we give some appropriate remarks. When the letter $P$ appears in this column, it indicates that one of $R_{1}$ or $R_{2}$ is a pseudoprime, even though this fact was not needed by the program to prove the corresponding $N_{n}$ or $\bar{N}_{n}$ prime.

8. Some Special Cases. In this section we discuss some of the more interesting of the numbers of Table 1.

For $N=N_{368}$, the number in the introduction, we have (using the notation of [10])

$$
M>6 \times 10^{43}, \quad M_{3}>10^{24}
$$

and consequently

$$
N<\min \left(M M_{3}, M_{3}^{3}\right)
$$


TABLE 1

\begin{tabular}{|c|c|c|c|c|}
\hline $\mathrm{N}$ & $\begin{array}{l}\text { No. of } \\
\text { Digits }\end{array}$ & B & Tests & Remarks \\
\hline $\mathrm{N}_{206}$ & 38 & & {$[1],[10], \mathrm{PW}$} & $\mathrm{P}$ \\
\hline $\mathrm{N}_{208}$ & 29 & & {$[1],[10]$} & $\mathrm{P}$ \\
\hline $\mathrm{N}_{212}$ & 36 & & {$[1],[10], \mathrm{PW}$} & $\mathrm{P}$ \\
\hline $\mathrm{N}_{218}$ & 42 & & {$[1],[10]$} & $\begin{array}{l}\text { Pollard's method found the factor } \\
8570437 \text { of } \mathrm{N}+1 \text {. }\end{array}$ \\
\hline$\overline{\mathrm{N}}_{223}$ & 30 & & {$[1],[10]$} & \\
\hline $\mathrm{N}_{229}$ & 45 & & {$[1],[10], \mathrm{PW}$} & $\begin{array}{l}\text { Pollard's method found the factor } \\
2948041 \text { of } \mathrm{N}+1 \text {. }\end{array}$ \\
\hline$N_{239}$ & 44 & & {$[1],[10]$} & $\mathrm{P}$ \\
\hline $\mathrm{N}_{241}$ & 38 & & {$[1],[10]$} & $P$ \\
\hline $\mathrm{N}_{247}$ & 31 & & {$[1],[10]$} & $P$ \\
\hline $\mathrm{N}_{259}$ & 42 & & {$[1],[10]$} & $\mathbf{P}$ \\
\hline $\mathrm{N}_{263}$ & 40 & & {$[1],[10]$} & \\
\hline $\bar{N}_{263}$ & 46 & & {$[1],[10], \mathrm{PW}$} & $\mathrm{P}$ \\
\hline$N_{278}$ & 53 & & {$[1],[10], \mathrm{PW}$} & $\mathbf{P}$ \\
\hline$\overline{\mathrm{N}}_{281}$ & 46 & & {$[1],[10]$} & $\mathrm{P}$ \\
\hline$N_{289}$ & 48 & $1.6 \times 10^{7}$ & {$[1],[10], \mathrm{PW}$} & \\
\hline $\mathrm{N}_{293}$ & 56 & & {$[1],[10]$} & \\
\hline $\bar{N}_{299}$ & 56 & & {$[1],[10]$} & \\
\hline $\mathrm{N}_{307}$ & 62 & $3 \times 10^{8}$ & {$[1],[10], \mathrm{PW}$} & See discussion below. \\
\hline $\mathrm{N}_{311}$ & 61 & & {$[1],[10], \mathrm{PW}$} & \\
\hline$\overline{\mathrm{N}}_{311}$ & 44 & & {$[1],[10], \mathrm{PW}$} & $\mathrm{P}$ \\
\hline $\mathrm{N}_{314}$ & 57 & & {$[1],[10], \mathrm{PW}$} & \\
\hline $\mathrm{N}_{316}$ & 60 & $1.1 \times 10^{6}$ & {$[1],[10], \mathrm{PW}$} & $\begin{array}{l}\mathrm{R}_{1} \text { (53 digits) proved prime using } \\
{[1],[7], \mathrm{PW}(\mathrm{P}) \text {, then iN proved }} \\
\text { prime using [1]. }\end{array}$ \\
\hline $\mathrm{N}_{319}$ & 50 & & {$[1],[10], \mathrm{PW}$} & 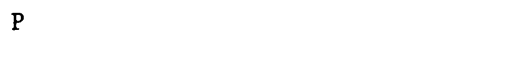 \\
\hline $\mathrm{N}_{321}$ & 36 & & [1] & $\begin{array}{l}\mathrm{R}_{2} \text { proved piime, then } \mathrm{N} \text { proved } \\
\text { prime. }\end{array}$ \\
\hline$\overline{\mathrm{N}}_{323}$ & 51 & $2.5 \times 10^{6}$ & {$[1],[10], \mathrm{PW}$} & \\
\hline $\mathrm{N}_{332}$ & 64 & $6 \times 10^{7}$ & [1] & $\begin{array}{l}\mathrm{N}+1=2^{3} \cdot 3 \cdot 7 \cdot 83 \cdot 59138939 \cdot \mathrm{R} 2 . \\
\mathrm{R}_{2} \text { proved prime, then } \mathrm{N} \text { proved } \\
\text { prime. }\end{array}$ \\
\hline$N_{337}$ & 58 & & [1] & $\begin{array}{l}\text { Pollard's method found the factor } \\
12815681 \text { of } \mathrm{N}-1 \text {. }\end{array}$ \\
\hline
\end{tabular}


TABLE 1 (continued)

\begin{tabular}{|c|c|c|c|c|}
\hline $\mathrm{N}$ & $\begin{array}{l}\text { No, of } \\
\text { Digits }\end{array}$ & B & Tests & Remarks \\
\hline$N_{341}$ & 50 & & {$[1],[10]$} & $P$ \\
\hline$N_{343}$ & 46 & & [1] & $\begin{array}{l}\text { Here } R_{2}-1=F_{1}^{\prime} R_{1}^{\prime} \cdot \quad R_{1}^{\prime} \text {, then } R_{2} \text {, } \\
\text { then } N \text { proved prime. }\end{array}$ \\
\hline $\mathrm{N}_{356}$ & 69 & & {$[1],[10], \mathrm{PW}$} & See discussion below. \\
\hline $\mathrm{N}_{357}$ & 41 & & {$[1],[10]$} & $\begin{array}{l}\text { Pollard's method found the factor } \\
106929516613 \text { of } \mathrm{N}^{2}+1 .\end{array}$ \\
\hline$N_{368}$ & 62 & $1.8 \times 10^{6}$ & {$[1],[10], \mathrm{PW}$} & See discussion below. \\
\hline$\overline{\mathrm{N}}_{371}$ & 61 & $5 \times 10^{7}$ & {$[1],[10], \mathrm{PW}$} & See discussion below. \\
\hline $\mathrm{N}_{372,}$ & 47 & & {$[1],[10], \mathrm{PW}$} & $\mathrm{P}$ \\
\hline$N_{373}$ & 75 & & {$[1],[10], \mathrm{PW}$} & $P$ \\
\hline$\overline{\mathrm{N}}_{373}$ & 58 & & {$[1],[10]$} & $P$ \\
\hline $\mathrm{N}_{381}$ & 43 & & {$[1],[10], \mathrm{PW}$} & \\
\hline
\end{tabular}

Thus, $N=p_{1} p_{2}$, where $p_{1}$ and $p_{2}$ are primes,

$$
p_{1} \equiv 762385150126634192052929\left(\bmod F_{1} \bar{F}_{2} \bar{F}_{4}\right), \quad p_{2}>10^{24} \text {, }
$$

and $\left(C^{2}-16 D \mid p_{1}\right)=-1,\left(D \mid p_{1}\right)=-1$. By verifying (3) and using (b) for $p_{1}$, it was shown by actual division that any possible value of $p_{1}$ less than $F_{1} \bar{F}_{2} \bar{F}_{4} \bar{F}_{6}$ is not a divisor of $N$. Hence

$$
p_{1}>9 \times 10^{23} \times 2.7 \times 10^{13}>2.4 \times 10^{37} \text { and } p_{1} p_{2}>2.4 \times 10^{61}>N
$$

Thus $N$ must be a prime.

\section{For}

$N_{356}=565768471959285714079262248889509474547974219027885983055827845016103$

and $B=5 \times 10^{5}$, we get

$$
\begin{gathered}
F_{1}=2 \cdot 3 \cdot 659 \cdot 1567, \quad F_{2}=2^{3} \cdot 53 \cdot 89, \quad F_{4}=2 \cdot 5, \\
F_{3}=3 \cdot 7 \cdot 2659, \quad F_{6}=241 \cdot 7759 .
\end{gathered}
$$

This is not enough to prove $N_{356}$ a prime. However, $R_{1}$ and $R_{2}$ are both pseudoprime. The larger of these $\left(R_{2}\right)$ is easier to prove prime than $R_{1}$. In fact, if $N^{\prime}=R_{2}=14992804535702928611386004051555794852341907436609232114050981689$

(65 digits) and $B=3 \times 10^{6}$, we get

$$
\begin{gathered}
F_{1}^{\prime}=2^{3} \cdot 3 \cdot 67 \cdot 7411 \cdot 51169, \quad F_{2}^{\prime}=2 \cdot 5 \cdot 31 \cdot 23039 \\
F_{4}^{\prime}=2 \cdot 17^{2} \cdot 37, \quad F_{3}^{\prime}=3 \cdot 7 \cdot 13 \cdot 43 \cdot 61 \cdot 1087 \cdot 5119 \cdot 10501, \quad F_{6}^{\prime}=2221 .
\end{gathered}
$$


By using the tests of [1], [10] and PW (neither $R_{1}^{\prime}$ nor $R_{2}^{\prime}$ is a pseudoprime), $N^{\prime}$ can be shown to be a prime. It is then a simple matter to prove $N_{356}$ a prime.

For

$$
N=\bar{N}_{371}=1066891454330692360911118469915492770211286402568532457966113
$$

and $B=5 \times 10^{7}$, we get

$$
\begin{aligned}
& F_{1}=2^{5} \cdot 29, \quad F_{2}=2 \cdot 3 \cdot 7 \cdot 11 \cdot 53 \cdot 11239>2 \cdot 7 \times 10^{8}, \\
& F_{4}=2 \cdot 5 \cdot 165041, \quad F_{3}=1431907, \quad F_{6}=3 \cdot 13 \cdot 19,
\end{aligned}
$$

and $R_{1}$ and $R_{2}$ are both composite. With this information it is possible to demonstrate with the computer program that either $N$ is a prime or it is the product of two primes $p_{1}$ and $p_{2}$. Further, if $N=p_{1} p_{2}, p_{1}$ is a prime of the first kind; and $p_{2}$ is a prime of the second kind.

It follows by using the results of Section 6 that

$$
p_{1}=r_{1}, r_{1}^{\prime}, r_{1}^{\prime \prime}(\bmod K), \quad p_{2} \equiv r_{2}, r_{2}^{\prime}, r_{2}^{\prime \prime} \quad(\bmod K),
$$

where $r_{1}, r_{2}$ are obtained by using the first case of (a) and (b), $r_{1}^{\prime}, r_{2}^{\prime}$ by the second case of (a), (b), and $r_{1}^{\prime \prime}, r_{2}^{\prime \prime}$ by the third case of (a), (b).

Now $K=F_{1} \bar{F}_{2} \bar{F}_{3} \bar{F}_{4} \bar{F}_{6}>3 \cdot 7 \times 10^{25}$ and if

$$
p_{1}=r_{1}+t_{2} K, \quad p_{2}=r_{2}+t_{2} K,
$$

then

$$
N=r_{1} r_{2}+\left(t_{1} r_{2}+t_{2} r_{1}\right) K+t_{1} t_{2} K^{2}
$$

We now make use of an extension of an idea introduced in [1]. For if

$$
\left(N-r_{1} r_{2}\right) / K \equiv T\left(\bmod F_{2}\right)
$$

where $|T|<\bar{F}_{2}$, then, recalling that $r_{1} \equiv-1\left(\bmod F_{2}\right), r_{2} \equiv 1\left(\bmod F_{2}\right)$, we get

$$
t_{1}-t_{2} \equiv T\left(\bmod F_{2}\right) \text {. }
$$

If $t_{1}-t_{2} \neq T$, then $\left|t_{1}-t_{2}\right|>\bar{F}_{2}$; and consequently, $t_{1}$ or $t_{2}>\bar{F}_{2}>1.37 \times$ $10^{8}$. It is possible to verify on the computer that

$$
p_{1} \nvdash N, p_{2} \nmid N \text { for } 0 \leqslant t_{1}, t_{2} \leqslant 6 \text {; }
$$

hence

$$
p_{1} p_{2}>6 \times 1.37 \times(3.7)^{2} \times 10^{58}>N .
$$

If $t_{1}-t_{2}=T$, we must have

$$
A=\left(r_{1}-r_{2}+K T\right)^{2}+4 N
$$

a perfect integer square. It is easy to use the computer to find a small prime $\pi \nmid K$ such that $(A \mid \pi)=-1$. In a similar manner we can dispose of the other two cases: 
$p_{1} \equiv r_{1}^{\prime}, p_{2} \equiv r_{2}^{\prime}$ and $p_{1} \equiv r_{1}^{\prime \prime}, p_{2} \equiv r_{2}^{\prime \prime}(\bmod K)$. By using this strategy the number $N$ was proved prime.

For

$$
N=N_{307}=11739610117429203651282768407085324070169775523763828726810201
$$

and $B=3 \times 10^{8}$, we get

$$
\begin{gathered}
F_{1}=2^{3} \cdot 5^{2} \cdot 7 \cdot 307, \quad F_{2}=2 \cdot 3 \cdot 431 \cdot 6911>1.78 \times 10^{7} \\
F_{4}=2, \quad F_{3}=737497, \quad F_{6}=3 \cdot 229
\end{gathered}
$$

and $R_{1}, R_{2}$ are both composite. None of the other numbers considered in Table 1 presented as much difficulty in proving primality as this one. The strategy used to prove $N$ prime is a refinement of that used in demonstrating the primality of $\bar{N}_{371}$. In this particular example use was made of tests (1), (2), (3), and (4) as well as all the tests (I, II, III, IV) of [1] and the test $(\beta)$ of [10].

We have $M=1+B^{3} F_{1} \bar{F}_{2} \bar{F}_{4}>10^{38}$. In order to show that $N$ is the product of at most two primes, we must obtain a large $\left(>1.2 \times 10^{23}\right)$ lower bound on any prime of the first kind $p$ which divides $N$. We have

$$
p \equiv 1 \quad\left(q_{1} F_{1}\right), \quad p \equiv-1 \quad\left(q_{2} F_{2}\right)
$$

and if $(p \mid P)_{3}=1$,

$$
p \equiv 1 \quad\left(q_{3} F_{3}\right), \quad p \equiv-1 \quad\left(q_{6} F_{6}\right)
$$

where $q_{i}$ is some prime divisor of $R_{i}\left(q_{i}>B\right)$. In this case we have $p>1+B^{2} F_{1} \bar{F}_{3}$ $=2.5 \times 10^{28}>1.2 \times 10^{23}$. Since $\bar{F}_{3}$ and $\bar{F}_{6}$ are both primes, there are four possibilities for $p$ modulo $K\left(=F_{1} \bar{F}_{2} \bar{F}_{3} \bar{F}_{6}>6.48 \times 10^{20}\right)$ when $(p \mid P)_{3} \neq 1$. These are given by

$$
\begin{gathered}
\left\{\begin{array} { l l } 
{ p \equiv I i \quad ( \operatorname { m o d } \overline { F } _ { 3 } ) , } \\
{ p \equiv N \quad ( \operatorname { m o d } \overline { F } _ { 6 } ) , }
\end{array} \quad \left\{\begin{array}{l}
p \equiv-N-1 \quad\left(\bmod \bar{F}_{3}\right), \\
p \equiv N \quad\left(\bmod \bar{F}_{6}\right) .
\end{array}\right.\right. \\
\left\{\begin{array} { l l } 
{ p \equiv N \quad ( \operatorname { m o d } \overline { F } _ { 3 } ) , } \\
{ p \equiv - N + 1 \quad ( \operatorname { m o d } \overline { F } _ { 6 } ) , }
\end{array} \quad \left\{\begin{array}{ll}
p \equiv-N-1 \quad\left(\bmod \bar{F}_{3}\right), \\
p \equiv-N+1 \quad\left(\bmod \bar{F}_{6}\right) .
\end{array}\right.\right.
\end{gathered}
$$

We can obtain positive integers $A_{1}, A_{2}, A_{3}, A_{4}$ such that $p \equiv A_{i}(\bmod K)$ for some $i \leqslant 4\left(A_{i}<K\right)$. It was shown by machine that $A_{i}+t_{i} K \nmid N$ for $0 \leqslant t_{i} \leqslant 186$ and $1 \leqslant i \leqslant 4$; hence, if $p$ is a prime of the second kind and $p \mid N$, then

$$
p>186 \times 6.48 \times 10^{20}=1.2 \times 10^{23} \text {. }
$$

We have $M_{3}^{3}>N$ and $M_{3} M>N$; it follows that $N$ is prime or $N=p_{1} p_{2}$, where $p_{1}$ is a prime of the first kind and $p_{2}$ is a prime of the second kind. We suppose that 
$N$ is the product of two primes and deal with the three possible cases.

Case 1. $\left(p_{2} \mid P\right)_{3}=1$. We have

$$
p_{2} \equiv 1 \quad\left(q_{1} F_{1}\right), \quad p_{2} \equiv 1 \quad\left(q_{2} F_{2}\right), \quad p_{2} \equiv 1 \quad\left(q_{3} F_{3}\right), \quad p_{2} \equiv 1 \quad\left(q_{6} F_{6}\right)
$$

hence,

$$
p_{2}>1+B^{4} K>4 \times 10^{54} \text { and } p_{1} p_{2}>N
$$

Case 2. $\left(p_{1} \mid P\right)_{3}=1$. We have

$$
p_{1} \equiv 1 \quad\left(q_{1} F_{1}\right), \quad p_{2} \equiv 1 \quad\left(q_{1} q_{2} F_{1} \bar{F}_{2}\right), \quad p_{1} \equiv 1 \quad\left(q_{3} F_{3}\right)
$$

thus,

$$
p_{1}>1+B^{2} F_{1} \bar{F}_{3}>2.85 \times 10^{28}, \quad p_{2}>1+B^{2} F_{1} \bar{F}_{2}>3.45 \times 10^{29} .
$$

We also have

$$
\left\{\begin{array} { l l } 
{ p _ { 1 } \equiv - 1 } & { ( \operatorname { m o d } F _ { 2 } ) , } \\
{ p _ { 1 } \equiv - 1 } & { ( \operatorname { m o d } F _ { 6 } ) , }
\end{array} \quad \left\{\begin{array}{l}
p_{2} \equiv N \quad\left(\bmod F_{3}\right) \\
p_{2} \equiv-N \quad\left(\bmod F_{6}\right)
\end{array}\right.\right.
$$

Hence, we can determine integers $r_{1}, r_{2}$ such that

$$
p_{1} \equiv r_{1} \quad(\bmod K), \quad p_{2} \equiv r_{2} \quad(\bmod K) .
$$

If we use the argument employed in the discussion of $\bar{N}_{371}$, we see that for some $k$ we must have

$$
A(k)=\left(r_{1}-r_{2}+K T+k K F_{2}\right)^{2}+4 N
$$

a perfect square, where

$$
\begin{gathered}
T \equiv\left(N-r_{1} r_{2}\right) / K \quad\left(\bmod F_{2}\right), \quad|T|<\bar{F}_{2}, \\
p_{1}=r_{1}+t_{1} K, \quad p_{2}=r_{2}+t_{2} K, \quad t_{1}-t_{2}=T+k F_{2} .
\end{gathered}
$$

Let $\Pi$ be the set of all primes, which do not divide $K$ and are less than 100 . It was easily verified by using a sieve process that

$$
(A(k) \mid \pi)=-1 \quad \text { for some } \pi \in \Pi
$$

for each $k$ such that $0 \leqslant|k| \leqslant 3.6 \times 10^{4}$. Thus, since $\min \left(p_{1}, p_{2}\right)>2.85 \times 10^{28}$, and one of $t_{1}, t_{2}$ must exceed $(|k|-1 / 2) F_{2}$, we have

$$
p_{1} p_{2}>2.85 \times 10^{28} \times 6.48 \times 10^{20} \times 1.78 \times 10^{7} \times 3.6 \times 10^{4}>N .
$$

Case 3. $\left(p_{1} \mid P\right)_{3} \neq 1,\left(p_{2} \mid P\right)_{3} \neq 1$. We have

$$
-N-1 \equiv p_{1} \equiv p_{2} \quad\left(\bmod \bar{F}_{3}\right), \quad-N+1 \equiv p_{1} \equiv-p_{2} \quad\left(\bmod \bar{F}_{6}\right) .
$$

Hence, we can find $r_{1}, r_{2}$ (different from the preceding $r_{1}, r_{2}$ ) such that

$$
p_{1}=r_{1}+t_{1} K, \quad p_{2}=r_{2}+t_{2} K \text {. }
$$


Using reasoning similar to the above, we get

$$
t_{1}+t_{2} \equiv T \equiv r_{1}^{-1}\left(N-r_{1} r_{2}\right) / K \quad\left(\bmod F_{1} \bar{F}_{3}\right)
$$

where $|T|<\bar{F}_{1} \bar{F}_{3}$. We verified that

$$
A(k)=\left(r_{1}+r_{2}+K T+k K F_{1} \bar{F}_{3}\right)^{2}-4 N
$$

cannot be a perfect square for any $k$ such that $0 \leqslant k \leqslant 8.6 \times 10^{4}$, and we also verified that $r_{1}+t_{1} K \nmid N$ for $0 \leqslant t_{1} \leqslant 1040$.

Let

$$
t_{1}+t_{2}=2 a>(k-1 / 2) F_{1} \bar{F}_{3}>2.7 \times 10^{16}
$$

Since $p_{2}>3.45 \times 10^{29}$, it follows that $t_{1}<t_{2}$; hence,

$$
t_{1}=a-b, \quad t_{2}=a+b,
$$

where $0 \leqslant b \leqslant a$.

If $b>.999 a$, then $t_{2}>(1.999) a>2.698 \times 10^{16}$ and

$$
p_{1} p_{2}>1040 \times 6.48 \times 10^{20} \times 2.698 \times 10^{16} \times 6.48 \times 10^{20}>N .
$$

If $b \leqslant .999 a$, then

$$
t_{1} \geqslant(.001) a>1.35 \times 10^{13}, \quad t_{2} \geqslant a>1.35 \times 10^{16},
$$

and $p_{1} p_{2}>N$.

Since $N$ cannot be the product of two or more primes, it must be prime.

In conclusion, we remark that had we wished to use factors of $\left(N^{5}-1\right) /(N-1)$ or $\left(N^{5}+1\right) /(N+1)$ to prove the primality of $N_{307}$, we would find with $B=10^{6}$ that

$$
\begin{aligned}
& \left(N^{5}-1\right) /(N-1)=5 \cdot 11 \cdot 821 \cdot R_{5}, \\
& \left(N^{5}+1\right) /(N+1)=241 \cdot 9311 \cdot 9851 \cdot 35461 \cdot 151381 \cdot R_{10} .
\end{aligned}
$$

The unfortunate aspect of investigating factors of higher cyclotomic functions of $N$ is the very rapid proliferation of possible residue classes to which suspected prime divisors of $N$ could belong. For very large $N$ the creation of all the residue classes becomes so tedious and the resulting test divisions become so numerous that any advantage obtained by knowing a large collection of congruences that a suspected prime divisor must satisfy appears to be destroyed.

9. Acknowledgements. The authors gratefully acknowledge several suggestions from Professors John Brillhart and D. H. Lehmer. They also thank D. H. Lehmer for making available his notes [4] on primality testing. 
1. JOHN BRILLHART, D. H. LEHMER \& J. L. SELFRIDGE, "New primality criteria and factorizations of $2^{m} \pm 1$," Math. Comp., v. 29, 1975, pp. 620-647.

2. DOV JARDEN, Recurring Sequences, 3rd ed., Riveon Lemathematika, Jerusalem, 1973, pp. 41-59.

3. D. H. LEHMER, "The economics of number theoretic computation," Computers in Number Theory, Academic Press, London and New York, 1971, pp. 1-9. MR 47 \#3285.

4. D. H. LEHMER, "Use of Pierce functions for a primality test." (Unpublished notes.)

5. EMMA LEHMER, "Criteria for cubic and quartic residuacity," Mathematika, v. 5, 1958, pp. 20-29. MR $20 \# 1668$.

6 T. A. PIERCE, "The numerical factors of the arithmetic forms $\Pi^{n}\left(1 \pm \alpha_{i}^{m}\right)$," Ann. of Math. (2), v. 18, 1916, pp. 53-64.

7. J. M. POLLARD, "Theorems on factorization and primality testing," Proc. Cambridge Philos. Soc., v. 76, 1974, pp. 521-528. MR 50 \#6992.

8. J. L. SELFRIDGE \& M. C. WUNDERLICH, "An efficient algorithm for testing large numbers for primality," Proc. Fourth Manitoba Conf. on Numerical Math. (Winnipeg, Man.,1974), Congr. Numer., No. 12, Utilitas Math., Winnipeg, Man., 1975, pp. 109-120. MR 51 \#5461.

9. H. C. WILliAMS, “A generalization of Lehmer's functions," Acta Arith., v. 29, 1976, pp. 315-341.

10. H. C. WILLIAMS \& J. S. JUDD, "Determination of the primality of $N$ by using factors of $N^{2} \pm 1$," Math. Comp., v. 30, 1976, pp. 157-172.

11. M. C. WUNDERLICH \& J. L. SELFRIDGE, "A design for a number theory package with an optimized trial division routine," Comm. $A C M$, v. 17, 1974, pp. 272-276. 